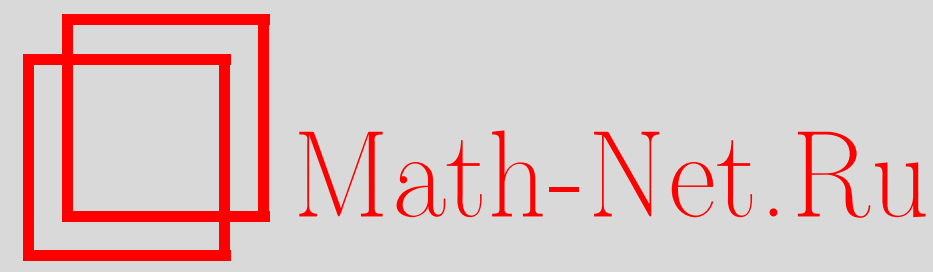

Н. Н. Холщевникова, K теореме Валле-Пуссена о единственности представления функции тригонометрическим рядом, Матем. сб., 1996, том 187, номер 5, 143-160

DOI: https://doi.org/10.4213/sm132

Использование Общероссийского математического портала Math-Net.Ru подразумевает, что вы прочитали и согласны с пользовательским соглашением http://www . mathnet.ru/rus/agreement

Параметры загрузки:

IP : 54.198 .187 .58

26 апреля 2023 г., 12:53:00 
УДК 517.5

\author{
Н.Н. Холщевникова
}

\title{
К теореме Валле-Пуссена о единственности представления функции тригонометрическим рядом
}

Теорема Валле-Пуссена говорит о том, что из сходимости тригонометрического ряда к конечной интегрируемой функции $f$ всюду за исключением счетного множества $E$ следует, что этот ряд есть ряд Фурье функции $f$. В работе показано, что теорема остается справедливой, если в качестве исключительного множества $E$ взять объединение счетного числа $H$-множеств.

Библиографиял: 7 названий.

Основным результатом о единственности представления функции тригонометрическим рядом является следующая

ТЕОРЕМА 1 ВАЛЛЕ-ПУССЕНА. Если за исключением точек счетного множсества Е тригонометрический ряд сходится к конечной и интегрируемой функиии $f$, то он является рядом Фурье этой функиии.

В 1923 г. И.И. Привалов доказал, в частности, что в этой теореме вместо счетного множества $E$ можно взять замкнутое множество единственности. Мы докажем, что в качестве исключительного множества $E$ в этой теореме 1 Валле-Пуссена можно взять объединение замкнутого множества единственности и счетного числа $H$-множеств. При условии ограниченности функции $f$ и пустого множества $E$ теорема Валле-Пуссена превращается в доказанную раньше теорему дю Буа-Реймона-Лебега. Если в условии теоремы Валле-Пуссена потребовать дополнительно ограниченность функции $f$, то мы покажем, что теорема остается справедливой, если в качестве исключительного множества $E$ взять множество единственности типа $F_{\sigma}$.

\section{§1. Множества единственности для метода суммирования Римана}

Будем рассматривать тригонометрические ряды

$$
\frac{a_{0}}{2}+\sum_{n=1}^{\infty} a_{n} \cos n x+b_{n} \sin n x
$$

с коэффициентами, стремящимися к нулю

$$
\lim _{n \rightarrow \infty} a_{n}=\lim _{n \rightarrow \infty} b_{n}=0
$$

Работа вьполнена при поддержке Российского фонда фундаменталњных исследований (грант № 94-01-00417). 
Функиией Римана для тригонометрического ряда (1), (2) называется функция

$$
F(x)=\frac{a_{0} x^{2}}{4}+A x+B-\sum_{n=1}^{\infty} \frac{a_{n} \cos n x+b_{n} \sin n x}{n^{2}}
$$

т.е. сумма дважды формально проинтегрированного ряда (1), (2).

Пусть $\Delta_{h}^{2} F(x)=F(x+h)+F(x-h)-2 F(x)$. Функция $F(x)$ называется гладкой в точке $x$, если

$$
\lim _{h \rightarrow 0} \frac{\Delta_{h}^{2} F(x)}{h}=0
$$

Известно, что функция Римана ряда (1), (2) является всюду гладкой.

Верхней и нижсей производными Шварца функции $F(x)$ называются, соответственно,

$$
\bar{D}^{2} F(x)=\varlimsup_{h \rightarrow 0} \frac{\Delta_{h}^{2} F(x)}{h^{2}} \text { и } \underline{D}^{2} F(x)=\varliminf_{h \rightarrow 0} \frac{\Delta_{h}^{2} F(x)}{h^{2}}
$$

(в каждой точке $x$, в окрестности которой определена функция $F(x)$, производные $\bar{D}^{2} F(x)$ и $\underline{D}^{2} F(x)$ сушествуют, конечные или бесконечные определенного знака).

Второй производной Швариа или второй симметрической производной функции $F(x)$ называется

$$
D^{2} F(x)=\lim _{h \rightarrow 0} \frac{\Delta_{h}^{2} F(x)}{h^{2}}
$$

Числовой ряд $\sum_{n=0}^{\infty} u_{n}$ суммируется методом Римана к $s$, если

$$
\lim _{h \rightarrow 0}\left[u_{0}+\sum_{n=1}^{\infty} u_{n}\left(\frac{\sin n h}{n h}\right)^{2}\right]=s
$$

Метод Римана является регулярньм методом суммирования, т.е. из сходимости числового ряда к конечному числу $s$ следует его суммируемость методом Римана к этому числу $s$. Так как для функции Римана $F(x)$ ряда $(1),(2)$ справедливо равенство

$$
\frac{\Delta_{2 h}^{2} F(x)}{4 h^{2}}=\frac{a_{0}}{2}+\sum_{n=1}^{\infty}\left(a_{n} \cos n x+b_{n} \sin n x\right)\left(\frac{\sin n h}{n h}\right)^{2}
$$

то суммируемость ряда (1), (2) в точке $x$ методом Римана эквивалентна существованию $D^{2} F(x)$.

Теперь может быть сформулирована в полной общности 
ТЕОРЕМа 2 ВАЛЛЕ-ПУССЕНА. Если для тригонометрического ряда $S$ вида (1), (2) всюду на $[0,2 \pi)$ за исключением, быть может, счетного множества Е выполняется одно из условий (9)-(11):

$$
\begin{gathered}
\underset{n \rightarrow \infty}{\lim _{n \rightarrow \infty}} s_{n}(x) \text { u } \varlimsup_{n \rightarrow \infty} s_{n}(x) \text { конечны на }[0,2 \pi) \backslash E \text { и интегрируемы, } \\
\text { әде } s_{n}(x)=\frac{a_{0}}{2}+\sum_{k=1}^{n} a_{k} \cos k x+b_{k} \sin k x ; \\
D^{2} F(x)=f(x), \quad x \in[0,2 \pi) \backslash E,
\end{gathered}
$$

или

$$
\underline{D}^{2} F(x) \leqslant f(x) \leqslant \bar{D}^{2} F(x), \quad x \in[0,2 \pi) \backslash E,
$$

где $F(x)$ - функция Римана ряда (1), (2), а $f(x)$ - конечная интегрируемая функиия,

то $D^{2} F(x)$ существует почти всюду, интегрируема, и $S$ есть ее ряд Фурье.

В этой теореме условие (11) самое слабое.

Напомним определения.

Множество $E \subset[0,2 \pi)$ называется множеством единственности или $\mathscr{U}$-множеством для тригонометрических рядов, если из сходимости ряда вида (1) к нулю на $[0,2 \pi) \backslash E$ следует, что все коэффициенты ряда равны нулю.

Множество $E \subset[0,2 \pi)$ назьвается множеством единственности для метода суммирования Римана или $\mathscr{U}(R)$-множеством, если из суммируемости методом Римана тригонометрического ряда (1), (2) к нулю на $[0,2 \pi) \backslash E$ следует, что все коэффициенты ряда равны нулю.

Всякое $\mathscr{U}(R)$-множество является $\mathscr{U}$-множеством. Известно, что счетное множество и замкнутое $\mathscr{U}$-множество являются $\mathscr{U}(R)$-множествами. Для счетного множества этот результат следует из теоремы 2 Валле-Пуссена (с условием (10)). Для замкнутого $\mathscr{U}$-множества это следует из того, что суммируемость методом Римана ряда $(1),(2)$ к нулю на интервале $(a, b)$ влечет сходимость этого ряда к нулю на $(a, b)$ (см. $[1$, c. 197]). Является ли произвольное $\mathscr{U}$-множество также и $\mathscr{U}(R)$-множеством неизвестно.

Если тригонометрический ряд (1), (2) почти всюду, но не всюду, суммируется методом Римана к нулю, то назовем его нуль-рядом для метода Римана или $R$-нуль-рядом.

Ядром $R$-нуль-ряда назовем множество точек, где он не суммируется методом Римана к нулю.

Приведенным ядром $R$-нуль-ряда назовем множество точек, где $\underline{D}^{2} F(x)$ или $\bar{D}^{2} F(x)$ бесконечны. Тогда приведенное ядро - это множество

$$
\mathscr{N}=[0,2 \pi) \backslash\left\{x:-\infty<\underline{D}^{2} F(x) \leqslant \bar{D}^{2} F(x)<+\infty\right\} .
$$

Сверхприведенным ядром назовем множество $\mathscr{N}_{1}$ всех тех точек $x$ из $[0,2 \pi)$, в которых

$$
D^{2} F(x)=+\infty \text { или } D^{2} F(x)=-\infty .
$$


Покажем, что $\mathscr{N}_{\text {и }} \mathscr{N}_{1}-$ несчетные борелевские множества типа $G_{\delta}$ и $F_{\sigma \delta}$, соответственно.

Справедлива следующая

Лемма 1. Пусть $F(x)$ - непрерывная функиия на интервале $(a, b)$. Тогда множество точек

$$
\mathscr{E}=\left\{x \in(a, b):-\infty<\underline{D}^{2} F(x) \leqslant \bar{D}^{2} F(x)<+\infty\right\}
$$

имеет тип $F_{\sigma}$, а множество $\mathscr{N}_{1}=E_{+} \cup E_{-}$, где

$$
E_{+}=\left\{x: D^{2} F(x)=+\infty\right\}, \quad E_{-}=\left\{x: D^{2} F(x)=-\infty\right\},
$$

uмeет mun $F_{\sigma \delta}$.

ДокАЗАТЕЛьство. Заметим, что $\mathscr{E}=\bigcup_{m=1}^{\infty} E_{m}$, где

$$
E_{m}=\left\{x \in(a, b):-m \leqslant \underline{D}^{2} F(x) \leqslant \bar{D}^{2} F(x) \leqslant m\right\} .
$$

Положим

$$
E_{m k}=\left\{x \in(a, b):\left|\Delta_{h}^{2} F(x)\right| \leqslant m h^{2} \text { при }|h| \leqslant 1 / k\right\} .
$$

Очевидны включения

$$
E_{m k} \subset E_{m} \quad(k=1,2,3, \ldots), \quad \bigcup_{k=1}^{\infty} E_{m k} \subset E_{m}
$$

и

$$
E_{m} \subset \bigcup_{k=1}^{\infty} E_{m+1, k}
$$

Так как

и

$$
\bigcup_{m=1}^{\infty} \bigcup_{k=1}^{\infty} E_{m k} \subset \mathscr{E}=\bigcup_{m=1}^{\infty} E_{m} \subset \bigcup_{m=1}^{\infty} \bigcup_{k=1}^{\infty} E_{m+1, k}
$$

TO

$$
\bigcup_{k=1}^{\infty} E_{m k} \supset \bigcup_{k=1}^{\infty} E_{m+1, k}
$$

$$
\mathscr{E}=\bigcup_{m=1}^{\infty} \bigcup_{k=1}^{\infty} E_{m k}
$$

Множества $E_{m k}$ замкнуты в силу непрерывности функции $F(x)$, следовательно, $\mathscr{E}$ имеет тип $F_{\sigma}$.

Обратимся теперь к множеству $\mathscr{N}_{1}$. В силу определения $E_{+}, x \in E_{+}$тогда и только тогда, когда для всякого $m>0$ найдется номер $k$ такой, что

$$
\Delta_{h}^{2} F(x) \geqslant m h^{2} \text { при }|h| \leqslant \frac{1}{k},
$$

т.е. $E_{+}=\bigcap_{m=1}^{\infty} \bigcup_{k=1}^{\infty} F_{k m}$, где множества

$$
F_{k m}=\left\{x \in(a, b): \Delta_{h}^{2} F(x) \geqslant m h^{2} \text { при }|h| \leqslant 1 / k\right\}
$$

замкнуты. Следовательно, $E_{+} \in F_{\sigma \delta}$ и, аналогично, $E_{-} \in F_{\sigma \delta}$. Тогда и $\mathscr{N}_{1}$ типа $F_{\sigma \delta}$, и лемма доказана.

Из этой леммы следует 
ЛЕмма 2. Приведенное ядро $\mathscr{N}$ и сверхприведенное ядро $\mathscr{N}_{1} \partial \Omega я ~ R$-нуль-ряда являются несчетными множествами типа $G_{\delta}$ и $F_{\sigma \delta}$, соответственно.

ДокАЗАтЕльство. Пусть $S$ - нуль-ряд для метода Римана и $F(x)$ - функция Римана для $S$. Как известно, $F(x)$ - непрерывная функция, и в силу леммы 1 сверхприведенное ядро $\mathscr{N}_{1}$ ряда $S$ будет типа $F_{\sigma \delta}$, а приведенное ядро, ввиду $(12),-$ типа $G_{\delta}$.

Допустим, что $\mathscr{N}_{1}$ счетно. Положим

$f(x)=\bar{D}^{2} F(x)$ там, где $\bar{D}^{2} F(x)$ конечна, и

$f(x)=\underline{D}^{2} F(x)$ там, где $\bar{D}^{2} F(x)=+\infty$, а $\underline{D}^{2} F(x)$ конечна.

Тогда функция $f(x)$ определена на $[0,2 \pi) \backslash \mathscr{N}_{1}$, для ряда $S$ выполняются условия теоремы 2 Валле-Пуссена с условием (11), и он есть ряд Фурье функции $f(x)$. Но так как $S$ - нуль-ряд для метода Римана, то $f(x)$ почти всюду равна нулю. Отсюда следует, что $S$ - тождественно нулевой ряд. Полученное противоречие доказывает, что множество $\mathscr{N}_{1}$, а значит, и $\mathscr{N} \supset \mathscr{N}_{1}$ несчетны. Лемма доказана.

Формальным произведением ряда (1), который в комплексной форме записывается в виде

$$
\sum_{n=-\infty}^{\infty} c_{n} e^{i n x}, \text { где } c_{-n}=\bar{c}_{n}, \quad c_{n}=\frac{a_{n}-i b_{n}}{2},
$$

и тригонометрического ряда

$$
\sum_{n=-\infty}^{\infty} \gamma_{n} e^{i n x}
$$

называется ряд

$$
\sum_{n=-\infty}^{\infty} K_{n} e^{i n x}, \text { где } K_{n}=\sum_{p=-\infty}^{\infty} c_{p} \gamma_{n-p} .
$$

Известно (результаты А. Райхмана, см., например, [1, с. 194]), что если $c_{n} \rightarrow 0$ и ряд $\sum\left|\gamma_{n}\right|$ сходится, то $K_{n} \rightarrow 0$. При этом ряд (14) является рядом Фурье своей суммы $\lambda(x)$. Если $c_{n} \rightarrow 0$ и ряд $\sum\left|n \gamma_{n}\right|$ сходится, то ряд

$$
\sum_{n=-\infty}^{\infty}\left(K_{n}-\lambda(x) c_{n}\right) e^{i n x}=\sum_{n=-\infty}^{\infty} K_{n} e^{i n x}-\lambda(x) \sum_{n=-\infty}^{\infty} c_{n} e^{i n x}
$$

сходится к нулю равномерно на $[0,2 \pi)$.

Условие $\sum\left|n \gamma_{n}\right|<\infty$ выполняется, в частности, если $\sum \gamma_{n} e^{i n x}$ есть ряд Фурье функции $\lambda(x)$, имеюшей три непрерьвные производные.

Лемма 3. Пусть $S$ - ряд вида (1), (2), а ряд $\sigma$ является формальным произведением (15) ряда $S$ и ряда (14), удовлетворяющего условию $\sum\left|n \gamma_{n}\right|<\infty$. Если функиия $\lambda(x)=\sum \gamma_{n} e^{\text {inx }}$ неотричательна, то для функиий Римана $F(x)$ и $\Phi(x)$ рядов $S$ и $\sigma$ выполняются равенства

$$
\underline{D}^{2} \Phi(x)=\lambda(x) \underline{D}^{2} F(x) \quad \text { u } \quad \bar{D}^{2} \Phi(x)=\lambda(x) \bar{D}^{2} F(x),
$$

в частности,

$$
D^{2} \Phi(x)=0, \quad \text { maм, где } \quad \lambda(x)=0 .
$$


ДоКАЗАТЕЛЬСТво. Из условия леммы следует, что ряд (16) сходится, а значит, и суммируется методом Римана к нулю на $[0,2 \pi)$. Из равенства (8) для функции $F(x)$ и аналогичного равенства для функции $\Phi(x)$ следует, что суммируемость методом Римана к нулю ряда $(16) \sigma-\lambda(x) S$ эквивалентна равенству

$$
\lim _{h \rightarrow 0}\left(\frac{\Delta_{2 h}^{2} \Phi(x)}{4 h^{2}}-\lambda(x) \frac{\Delta_{2 h}^{2} F(x)}{4 h^{2}}\right)=0 .
$$

Отсюда вытекают равенства (17) и (18). Лемма доказана.

Следствием леммы 3 является

ЛЕмма 4. Пусть $S$ является $R$-нуль-рядом с ядром $\mathscr{E}$, приведенныцм ядром $\mathscr{N}$ и сверхприведенныц ядром $\mathscr{N}_{1} . E c л и(a, b) \cap \mathscr{E} \neq \varnothing u(a, b) \subset[0,2 \pi)$, то найдется $R$-нуль-ряд $\sigma$, для которого ядром, приведенным ядром и сверхприведенным ядром служат множества $\mathscr{E} \cap(a, b), \mathscr{N} \cap(a, b)$ u $\mathscr{N}_{1} \cap(a, b)$, соответственно.

ДокАЗАтЕльство. Пусть $\lambda(x)$ - функция, равная нулю на $[0,2 \pi) \backslash(a, b)$, положительная на $(a, b)$ и имеющая непрерывные производные до третьего порядка включительно. Тогда $\lambda(x)=\sum \gamma_{n} e^{i n x}$, где $\sum\left|n \gamma_{n}\right|<\infty$. Рассмотрим ряд $\sigma$, являюшийся формальным произведением ряда $S$ на ряд Фурье функции $\lambda(x)$. В силу леммы 3 ряд $\sigma$ суммируется методом Римана к нулю на $[0,2 \pi) \backslash(a, b)$, так как там $\lambda(x)=0$, а так как $\lambda(x)>0$ на $(a, b)$ и ограничена, то из равенств (17) следует, что $\sigma$ есть $R$-нуль-ряд с ядром $\mathscr{E} \cap(a, b)$, приведенным ядром $\mathscr{N} \cap(a, b)$ и сверхприведенным ядром $\mathscr{N}_{1} \cap(a, b)$. Лемма доказана.

И, наконец, завершает данный ряд лемм

ЛЕмма 5. Пусть $S$ есть $R$-нуль-ряд с ядром $\mathscr{E}$ и приведеннбцм ядром $\mathscr{N}, a$ $E$ - множество единственности типа $F_{\sigma}$. Тогда $\mathscr{N} \backslash E \neq \varnothing$.

ДоказАТЕЛЬСтво. Допустим, что $\mathscr{N} \subset E$, где $E=\bigcup_{k=1}^{\infty} E_{k}$ и $E_{k}-$ замкнутые множества единственности. Тогда $\mathscr{N}=\bigcup_{k=1}^{\infty}\left(\mathscr{N} \cap E_{k}\right)$ и так как $\mathscr{N}$ имеет тип $G_{\delta}$, то одно из множеств $\mathcal{N} \cap E_{k}$ плотно в некоторой непустой порции множества $\mathscr{N}$ - множестве $\mathscr{N} \cap(a, b)$ для $(a, b) \subset[0,2 \pi)$. Так как $E_{k}$ замкнуто, то

$$
\mathscr{N} \cap(a, b) \subset E_{k} \cap(a, b) .
$$

В силу леммы 4 найдется $R$-нуль-ряд $\sigma$ с ядром $\mathscr{E} \cap(a, b)$ и приведенным ядром $\mathscr{N} \cap(a, b)$. Так как $E_{k}$ - замкнутое $\mathscr{U}$-множество, то $E_{k}$ является также $\mathscr{U}(R)$-множеством. Поэтому ряд $\sigma$ имеет точку ядра $x$, лежашую на $(a, b)$ вне $E_{k}$. Так как $E_{k}$ замкнуто, то найдется интервал $(\alpha, \beta) \subset(a, b)$ такой, что $x \in(\alpha, \beta),(\alpha, \beta) \cap E_{k}=\varnothing$. Так как $x \in \mathscr{E} \cap(a, b) \neq \varnothing$, то в силу леммы 4 найдется $R$-нуль-ряд $\sigma_{1}$ с ядром $\mathscr{E} \cap(\alpha, \beta)$ и приведенным ядром $\mathscr{N} \cap(\alpha, \beta)$. Но $\mathscr{N} \cap(\alpha, \beta) \subset E_{k} \cap(\alpha, \beta)=\varnothing$. С другой стороны, в силу леммы 2 приведенное ядро $R$-нуль-ряда непусто. Полученное противоречие доказьвает, что $\mathscr{N} \backslash E \neq \varnothing$ для всякого $\mathscr{U}$-множества $E$ типа $F_{\sigma}$. Лемма доказана.

Из леммы 5 непосредственно следует обобщение известной теоремы Бари об объединении счетного числа замкнутых множеств единственности. 
ТЕОРема 1. Объединение счетного числа замкнутых множеств единственности является множеством единственности для метода Римана.

ДоказАтЕльство. Пусть $E=\bigcup_{k=1}^{\infty} E_{k}$, где $E_{k}$ - замкнутые множества единственности. Допустим, что $E$ не является $\mathscr{U}(R)$-множеством и $S$ - соответствующий ненулевой ряд вида $(1),(2)$, суммируемый методом Римана к нулю на $[0,2 \pi) \backslash E$. Так как мера множества $E$ равна нулю, то $S$ есть $R$-нуль-ряд.

Пусть $\mathscr{E}-$ ядро, а $\mathscr{N}$ - приведенное ядро ряда $S$. Тогда $\mathscr{N} \subset \mathscr{E} \subset E$, но это противоречит лемме 5. Теорема доказана.

Карлет и Дебс [2] доказали теорему, которую можно назвать

ОБОБШЕННАЯ ТЕОРЕМА БАРИ. Объединение счетного числа множеств единственности $E_{k}$, замкнутых относительно своего обвединения $E=$ $\bigcup_{k=1}^{\infty} E_{k}$, является множеством единственности.

Из этой теоремы вытекают следующие результаты об объединении $\mathscr{U}$-множеств, доказанные ранее в [3]:

обтединение произвольного $\mathscr{U}$-множсества и $\mathscr{U}$-множсества типа $F_{\sigma} u G_{\delta}$ одновременно является И-множеством;

обгединение двух непересекающихся $\mathscr{U}$-множеств типа $G_{\delta}$ является U-множеством.

Аналогично можно обобщить и теорему 1 и получить из нее следствия, касающиеся объединения $\mathscr{U}(R)$-множеств. Для этого нам понадобится частично перекрывающаяся с леммой 5

Лемма 6. Пусть $S$ есть $R$-нуль-ряд с ядром $\mathscr{E}$ и пусть $E=\bigcup_{k=1}^{\infty} E_{k}$, әде $E_{k}$ являются $\mathscr{U}(R)$-мнохсествами, причем $E_{k}$ замкнутьи относительно Е. Тогда $\mathscr{E} \backslash E \neq \varnothing$.

Доказательство леммы 6 вполне аналогично доказательству леммы 5 . Более слабое требование относительной замкнутости множеств $E_{k}$ в $E$ по сравнению с замкнутостью множеств $E_{k}$ в лемме 5 влечет и более слабое утверждение $\mathscr{E} \backslash E \neq \varnothing$ по сравнению с утверждением $\mathscr{N} \backslash E \neq \varnothing$ леммы 5 .

Из леммы 6 следует обобщающая теорему 1

ТЕОРЕма 2. Объединение счетного числа $\mathscr{U}(R)$-множеств $E_{k}$, замкнутых относительно своего обвединения $E=\bigcup_{k=1}^{\infty} E_{k}$, является $\mathscr{U}(R)$-множсеством.

Доказательство теоремы 2 опирается на лемму 6 аналогично тому, как доказательство теоремы 1 опирается на лемму 5.

СлеДСТВИЕ. 1) Если $E_{1}$ и $E_{2}$ являются $\mathscr{U}(R)$-множествами типа $G_{\delta} u$ $E_{1} \cap E_{2}=\varnothing$, то $E_{1} \cup E_{2}$ тоже является $\mathscr{U}(R)$-множеством.

2) Если $E_{1}$ является $\mathscr{U}(R)$-множеством, а $E_{2}$ есть $\mathscr{U}$-множество типа $F_{\sigma}$ и $G_{\delta}$ одновременно, то $E_{1} \cup E_{2}$ является $\mathscr{U}(R)$-мнохеством.

Доказательство 1) и 2) следует из того, что в обоих случаях $E_{1}$ и $E_{2}$ имеют тип $F_{\sigma}$ относительно своего объединения. 
Теперь мы обратимся к вопросу единственности представления функции тригонометрическим рядом. Обобшением теоремы дю Буа-Реймона-Лебега является следующая

ТЕОРема 3. Пусть тригонометрический ряд $S$ вида (1), (2) суммируется методом Римана к конечной интегрируемой функиии $f(x)$ на множестве $[0,2 \pi) \backslash E$, где E есть $\mathscr{U}$-множество типа $F_{\sigma}$. Если функиия $f$ существенно ограничена, то $S$ есть ряд Фурье $S(f)$ функции $f$.

ДоказАТЕЛЬСтво. Допустим, что $S \neq S(f)$ и рассмотрим ряд $S-S(f)$. Покажем, что этот ряд будет $R$-нуль-рядом. Действительно, ряд Фурье интегрируемой функции почти всюду к ней суммируется методом Римана и, значит, $S-S(f)$ почти всюду суммируется к нулю, но так как $S \neq S(f)$, то $S-S(f)$ не может всюду суммироваться к нулю. Следовательно, $S-S(f)$ является нуль-рядом для метода Римана. Пусть $F(x)$ и $\Phi(x)$ - функции Римана рядов $S$ и $S(f)$. Так как $f$ существенно ограничена, то $\underline{D}^{2} \Phi(x)$ и $\bar{D}^{2} \Phi(x)$ ограничены на $[0,2 \pi)$. Тогда $\underline{D}^{2}(F(x)-\Phi(x))$ и $\bar{D}^{2}(F(x)-\Phi(x))$ конечны на $[0,2 \pi) \backslash E$. Следовательно, приведенное ядро $\mathscr{N}$ нашего $R$-нуль-ряда $S-S(f)$ содержится в $E$. Но это противоречит лемме 5 . Следовательно, наше предположение неверно, и $S=S(f)$. Теорема доказана.

В данном направлении может быть указана также

ТЕОРема 4. Пусть тригонометрический ряд $S$ вида (1), (2) суммируется методом Римана $к$ конечной интегрируемой функиии $f(x)$ на множсестве $[0,2 \pi) \backslash E$, әде $E$ есть $\mathscr{U}$-множество типа $F_{\sigma}$. Если функция $f(x)$ является точной производной, то $S$ есть ряд Фурье $S(f)$ функиии $f$.

ДокАЗАтельство. Как известно, $S(f)$ суммируется методом Римана к $f(x)$ в каждой точке, где ее первообразная имеет производную, равную $f(x)$ (см. [1, с. 190, 191]). Таким образом, в нашем случае ряд $S(f)$ суммируется методом Римана к $f(x)$ на $[0,2 \pi)$. Следовательно, $S-S(f)$ суммируется методом Римана к нулю на $[0,2 \pi) \backslash E$. Но множество $E$ в силу теоремы 1 является $\mathscr{U}(R)$-множеством. Следовательно, $S=S(f)$. Теорема доказана.

Дальше мы освободимся от дополнительных ограничений на функцию $f$, но при более узком классе исключительных множеств $E$.

\section{§2. Обобщение теоремы Валле-Пуссена}

При доказательстве теоремы Валле-Пуссена важную роль играет

Теорема А (см. [1, с. 785]). Пусть $f(x)$ суммируема на $(a, b)$ и конечна всюду, кроме, быть мохет, точек некоторого счетного множества Е. Если $F(x)$ непрерывна на $(a, b)$ и гладкая в точках $E$, то при выполнении неравенств

$$
\underline{D}^{2} F(x) \leqslant f(x) \leqslant \bar{D}^{2} F(x)
$$

всюду вне $E$, имеем

$$
F(x)=\int_{a}^{x} d y \int_{a}^{y} f(t) d t+A x+B
$$


всюдуна $(a, b)$, где $A$ и В постоянньи.

Доказательство же самой теоремы А сушественно опирается на свойства выпуклых функций, которые устанавливает

Tеорема В (см. [1, с. 783]). Если $F(x)$ непрерьвна на $(a, b)$ и если $\bar{D}^{2} F(x) \geqslant 0$ всюду на $(a, b)$, кроме, быть может, некоторого счетного множества $E, \boldsymbol{\theta}$ точках которого она гладкая, то $F(x)$ выпукла на $(a, b)$. Аналогично, если $\underline{D}^{2} F(x) \leqslant 0$, кроме точек $E$, то $F(x)$ вогнута. Наконеи, если $D^{2} F(x)=0$, кроме точек $E$, и гладкая в точках $E$, то $F(x)$ линейна на $(a, b)$.

Отметим, что в теореме В и в дальнейших леммах и теоремах условия $\bar{D}^{2} F(x) \geqslant 0$ или $\bar{D}^{2} F(x)>-\infty\left(\underline{D}^{2} F(x) \geqslant 0\right.$ или $\left.\underline{D}^{2} F(x)>-\infty\right)$ включают случай $\bar{D}^{2} F(x)=+\infty\left(\underline{D}^{2} F(x)=+\infty\right.$, соответственно $)$.

Нельзя ли в теореме В исключительное счетное множество, которое, как известно, является множеством единственности, заменить, например, замкнутым множеством единственности? При тех же самых условиях на функцию $F(x)$, что в теореме В, нельзя. Это показывает следуюший

Пример. Рассмотрим канторовское троичное множество $E$, построенное на отрезке $[0,2 \pi]$. Как известно, $E$ является замкнутым множеством единственности. Пусть $K(x)$ - канторовская ступенчатая функция, т.е. возрастающая непрерывная функция, построенная на смежных к $E$ интервалах, но не всюду, такая, что $K(0)=0, K(\pi)=1 / 2, K(2 \pi)=1$. Положим

$$
f(x)= \begin{cases}K(x), & x \in[0, \pi] \\ K(2 \pi-x), & x \in(\pi, 2 \pi]\end{cases}
$$

Первообразная $F(x)$ функции $f(x)$ является непрерывной, всюду гладкой функцией, при этом

$$
D^{2} F(x)=0 \text { для } x \in[0,2 \pi) \backslash E .
$$

Однако $F(x)$ не является линейной функцией, так как ее производная не является постоянной.

Но как известно, замкнутое множество единственности $E$ является $\mathscr{U}(R)$-множеством. Поэтому, если $F(x)$ - функция Римана ряда $S$ вида $(1),(2)$ и

$$
D^{2} F(x)=0 \text { для } x \in[0,2 \pi) \backslash E,
$$

то $F(x)=A x+B$.

Нерешенным остается

Вопрос. Если $F(x)$ - функиия Римана ряда $S$ вида (1), (2) и $\bar{D}^{2} F(x) \geqslant 0$ на $(a, b) \backslash E$, где $E$ - замкнутое множество единственности, будет ли $F(x)$ выпукла на $(a, b)$ ?

Мы можем доказать, что ответ на вопрос положителен только для специальных замкнутых $\mathscr{U}$-множеств.

Напомним определения. 
Множество $E \subset[0,2 \pi)$ называется $H$-множсеством, если сушествуют последовательность целых чисел $\left\{n_{k}\right\}$ и два числа $\alpha$ и $\delta, 0 \leqslant \alpha<1$ и $0 \leqslant \delta<1$, такие, что

$$
0 \leqslant\left\{n_{k} \frac{x}{2 \pi}-\alpha\right\} \leqslant \delta \text { для } x \in E, \quad k=1,2,3, \ldots,
$$

где $\{t\}$ - дробная часть числа $t$.

Эти множества введены и исследовались А. Райхманом. Обобщением понятия $H$-множества является введенное И. И. Пятецким-Шапиро понятие $H^{(s)}$-множества.

Последовательности цельх чисел $\left\{n_{k}^{(1)}\right\},\left\{n_{k}^{(2)}\right\}, \ldots,\left\{n_{k}^{(s)}\right\}$ назьвают независимымми, если для любого вектора $\left(x_{1}, x_{2}, \ldots, x_{s}\right)$ с целочисленньми координатами $x_{i}$ и с $\sum_{i=1}^{s} x_{i}^{2}>0$ выполняется условие

$$
\lim _{k \rightarrow \infty}\left|n_{k}^{(1)} x_{1}+n_{k}^{(2)} x_{2}+\cdots+n_{k}^{(s)} x_{s}\right|=+\infty
$$

Множество $E \subset[0,2 \pi)$ называется множеством типа $H^{(s)}$, если найдутся $s$ независимых последовательностей $\left\{n_{k}^{(1)}\right\},\left\{n_{k}^{(2)}\right\}, \ldots,\left\{n_{k}^{(s)}\right\}$ и параллелепипед $\Delta$, лежащий в единичном кубе $s$-мерного евклидова пространства, такие, что

$$
\left(\left\{n_{k}^{(1)} \frac{x}{2 \pi}\right\},\left\{n_{k}^{(2)} \frac{x}{2 \pi}\right\}, \ldots,\left\{n_{k}^{(s)} \frac{x}{2 \pi}\right\}\right) \notin \Delta \text { для } x \in E, \quad k=1,2,3, \ldots
$$

При $s=1$ множество типа $H^{(s)}$ есть обычное $H$-множество.

Пятецкий-Шапиро [4] доказал для любого $s \geqslant 2$ существование $H^{(s)}$-множества, не содержащегося в объединении счетного числа $H^{(s-1)}$-множеств.

Замькания $H$ - и $H^{(s)}$-множеств, как видно из определений, сами являются $H$ и $H^{(s)}$-множествами, соответственно. Райхман доказал, что $H$-множества, а Пятецкий-Шапиро, что $H^{(s)}$-множества, являются множествами единственности.

Далее мы докажем ряд утверждений, в которых исключительными множествами будут выступать $H^{(s)}$-множества и счетные объединения таких множеств.

Лемма 7. Пусть $F(x)$ - функция Римана ряда $S$ вида (1), (2). Если $\bar{D}^{2} F(x) \geqslant 0$ для $x \in[0,2 \pi) \backslash E$, әде $E$ есть $H^{(s)}$-множсество, то функиия $F(x)$ выпуклая.

ДокАЗАтЕльство. Известно (см. [1, с. 813-815], что для множества $E$ типа $H^{(s)}$ можно построить последовательность функций

$$
\lambda_{n}(x)=\sum_{k=-\infty}^{\infty} \alpha_{k}^{(n)} e^{i k x}
$$

обладающую свойствами:

1) $\lambda_{n}(x)=0$ для $x \in E, \lambda_{n}(x) \geqslant 0$ на $[0,2 \pi)$;

2) $\sum_{k=-\infty}^{\infty}\left|k \alpha_{k}^{(n)}\right|<\infty$

3) $\sum_{k=-\infty}^{\infty}\left|\alpha_{k}^{(n)}\right|<C$, где $C-$ постоянное число;

4) $\lim _{n \rightarrow \infty} \alpha_{k}^{(n)}=0$ для $k \neq 0$ и $\lim _{n \rightarrow \infty} \alpha_{0}^{(n)}=1$. 
Рассмотрим формальные произведения рядов Фурье функций $\lambda_{n}(x)$ и ряда $S-$ ряды $\sigma_{n}=\sum_{m} K_{m}^{(n)} e^{i m x}$ (см. [15]). Пусть $F(x)$ - функция Римана ряда $S$, а $F_{n}(x)$ - функции Римана рядов $\sigma_{n}$. Ввиду свойства 1$)$, из леммы 3 следует, что

$$
\bar{D}^{2} F_{n}(x)=\lambda_{n}(x) \bar{D}^{2} F(x) \geqslant 0 \text { при } x \in[0,2 \pi) \backslash E
$$

и

$$
D^{2} F_{n}(x)=0 \text { на } E .
$$

То есть $\bar{D}^{2} F_{n}(x) \geqslant 0$ всюду на [0, $\left.2 \pi\right)$ и, следовательно, $F_{n}(x)$ - выпуклые функции. В силу леммы Райхмана (см. [1, с. 812]) из свойств 2)-4) следует, что

$$
\lim _{n \rightarrow \infty} K_{m}^{(n)}=c_{m}=\frac{a_{m}-i b_{m}}{2} \quad(m=0, \pm 1, \pm 2, \ldots) .
$$

Заметим также, что

$$
\left|K_{m}^{(n)}\right|=\left|\sum_{r=-\infty}^{\infty} \alpha_{r}^{(n)} c_{m-r}\right| \leqslant \max _{m}\left|c_{m}\right| \cdot \sum_{r}\left|\alpha_{r}^{(n)}\right| \leqslant \text { const } .
$$

Функции Римана рядов $\sigma_{n}$ и $S$ имеют вид

$$
F_{n}(x)=\frac{K_{0}^{(n)}}{2} x^{2}-\sum_{m \neq 0} \frac{K_{m}^{(n)} e^{i m x}}{m^{2}}+A_{n} x+B_{n}
$$

и

$$
F(x)=\frac{c_{0} x^{2}}{2}-\sum_{m \neq 0} \frac{c_{m} e^{i m x}}{m^{2}}+A x+B .
$$

Пусть $F_{n}^{0}(x)=F_{n}(x)-A_{n} x-B_{n}, F^{0}(x)=F(x)-A x-B$. Тогда ввиду (19) и (20)

$$
\left|F^{0}(x)-F_{n}^{0}(x)\right|=\left|\frac{c_{0}-K_{0}^{(n)}}{2} x^{2}+\sum_{m \neq 0} \frac{K_{m}^{(n)}-c_{m}}{m^{2}} e^{i m x}\right|
$$

стремится к нулю при $n \rightarrow \infty$.

Функции $F_{n}^{0}(x)$ выпуклы, так как отличаются от выпуклых функций $F_{n}(x)$ на линейные слагаемые, поэтому функция $F^{0}(x)$ выпукла как предел последовательности выпуклых функций $F_{n}^{0}(x)$. Тогда $F(x)$ тоже выпукла, так как отличается от $F^{0}(x)$ на линейную функцию. Лемма доказана.

Лемма 8. Пусть

$$
F(x)=\frac{a_{0} x^{2}}{4}+A x+B-\sum_{n=1}^{\infty} \frac{a_{n} \cos n x+b_{n} \sin n x}{n^{2}}
$$

$\lim _{n \rightarrow \infty} a_{n}=\lim _{n \rightarrow \infty} b_{n}=0 . E c л u \bar{D}^{2} F(x) \geqslant 0$ при $x \in(a, b) \backslash E$, где E есть $H^{(s)}$-множество, то $F(x)$ выпукла на $(a, b)$. 
ДокАЗАТЕЛЬСтво. Не ограничивая общности, можем считать, что $(a, b) \subset$ $[0,2 \pi)$. Пусть $0<\varepsilon<(b-a) / 2$. Возьмем функцию $\lambda(x)$, равную нулю на $[0,2 \pi) \backslash$ $(a, b)$, равную единице на $(a+\varepsilon, b-\varepsilon)$, положительную на $(a, b)$ и имеюшую три непрерывные производные. Пусть ряд $\sigma$ есть формальное произведение ряда Фурье функции $\lambda(x)$ и ряда

$$
S=\frac{a_{0}}{2}+\sum_{n=1}^{\infty} a_{n} \cos n x+b_{n} \sin n x
$$

а $\Phi(x)$ - функция Римана ряда $\sigma$. Тогда $F(x)$ является функцией Римана ряда $S$ и ввиду леммы 3 и свойств функции $\lambda(x)$

$$
\bar{D}^{2} \Phi(x)=\lambda(x) \bar{D}^{2} F(x) \geqslant 0 \text { на }[0,2 \pi) .
$$

Следовательно, $\Phi(x)$ - выпуклая функция. Заметим, что на $(a+\varepsilon, b-\varepsilon)$ функция $F(x)$ отличается от $\Phi(x)$ разве лишь на линейную функцию. Действительно, так как ряд $\sigma-\lambda(x) \cdot S$ сходится к нулю всюду (см. (16)), а на $(a+\varepsilon, b-\varepsilon)$ это означает, что $\sigma-S$ сходится к нулю, а, значит, и суммируется к нулю методом Римана, то функция Римана ряда $\sigma-S$, равная $\Phi(x)-F(x)$, линейна на $(a+\varepsilon, b-\varepsilon)$. Следовательно, $F(x)$ вьпукла на $(a+\varepsilon, b-\varepsilon)$ и в силу произвольности $\varepsilon$ на $(a, b)$. Лемма доказана.

Лемма 9. Пусть $F(x)$ - функиия Римана ряда $S$ вида (1), (2). Если $\underline{D}^{2} F(x)>0$ при $x \in[0,2 \pi) \backslash E$, где $E=\bigcup_{k=1}^{\infty} E_{k}$ и $E_{k}$ являются $H^{\left(s_{k}\right)}$-множествами для некоторых натуральных $s_{k}$, то $F(x)$ - выпуклая функиия.

ДокаЗАТЕльство. Допустим, что $\left\{x: \underline{D}^{2} F(x)<0\right\} \neq \varnothing$. Заметим, что

$$
\left\{x: \underline{D}^{2} F(x)>0\right\} \subset \bigcup_{m=1}^{\infty} F_{m} \subset\left\{x: \underline{D}^{2} F(x) \geqslant 0\right\},
$$

где $F_{m}=\left\{x: \Delta_{h}^{2} F(x) \geqslant 0\right.$ при $\left.|h|<1 / m\right\}$ - замкнутые множества в силу непрерывности $F(x)$. Переходя к дополнениям этих множеств, получим

$$
\left\{x: \underline{D}^{2} F(x)<0\right\} \subset \bigcap_{m=1}^{\infty} G_{m} \subset\left\{x: \underline{D}^{2} F(x) \leqslant 0\right\},
$$

где $G_{m}=[0,2 \pi) \backslash F_{m}$ - открытые множества.

Положим

$$
G=\bigcup\left\{(\alpha, \beta):\left\{x: \underline{D}^{2} F(x)<0\right\} \cap(\alpha, \beta)=\varnothing\right\}
$$

и $\mathscr{E}=\bigcap_{m=1}^{\infty} G_{m} \backslash G$. Тогда $\mathscr{E}$ типа $G_{\delta}$, так как $G$ - открытое множество. Из условия леммы следует, что

$$
\mathscr{E} \subset E=\bigcup_{k=1}^{\infty} E_{k} .
$$

Так как замыкание $H^{\left(s_{k}\right)}$-множества является $H^{\left(s_{k}\right)}$-множеством, то, не ограничивая обшности, будем считать, что множества $E_{k}$ замкнуты. Так как $\mathscr{E}-$ множество 
типа $G_{\delta}$, то найдутся номер $k$ и интервал $(a, b)$ такие, что $\varnothing \neq(a, b) \cap E_{k} \cap \mathscr{E}$ плотно в $(a, b) \cap \mathscr{E}$ и в силу замкнутости $E_{k}$

$$
(a, b) \cap \mathscr{E} \subset(a, b) \cap E_{k} .
$$

Тогда $\underline{D}^{2} F(x) \geqslant 0$ на смежных к $E_{k}$ в $(a, b)$ интервалах, где $E_{k} \in H^{\left(s_{k}\right)}$. В силу леммы 8 отсюда следует, что функция $F(x)$ выпукла на $(a, b)$. Но тогда $\underline{D}^{2} F(x) \geqslant 0$ на $(a, b)$ и, следовательно, $(a, b) \subset G$. Это противоречит тому, что $\mathscr{E} \cap(a, b) \neq \varnothing$. Следовательно, предположение о том, что $\left\{x: \underline{D}^{2} F(x)<0\right\} \neq \varnothing$ приводит к противоречию. Значит, $\underline{D}^{2} F(x) \geqslant 0$ на $[0,2 \pi)$ и $F(x)$ вьпукла на $[0,2 \pi)$. Лемма доказана.

Лемма 10. Пусть $F(x)$ - функиия Римана ряда $S$ вида (1), (2). Если $\underline{D}^{2} F(x) \geqslant 0$ при $x \in(a, b) \backslash E$, где $E=\bigcup_{k=1}^{\infty} E_{k}, E_{k} \in H^{\left(s_{k}\right)}$, mо $F(x)$ вйпукла на $(a, b)$.

ДоказАТЕльСтво. Пусть сначала $(a, b)=(0,2 \pi)$ и $\underline{D}^{2} F(x) \geqslant 0$ на $(0,2 \pi) \backslash E$. Так как $F(x)$ является пределом функций $F_{n}(x)=F(x)+(1 / n) x^{2}$, a $\underline{D}^{2} F_{n}(x)=$ $\underline{D}^{2} F(x)+(2 / n)>0$ на $(0,2 \pi) \backslash E$, то в силу леммы 9 функции $F_{n}(x)$ выпуклы на $(0,2 \pi)$, а следовательно, и $F(x)$ выпукла на $(0,2 \pi)$.

Случай $\underline{D}^{2} F(x) \geqslant 0$ на $(a, b) \backslash E$ сводится к предыдущему умножением на функцию $\lambda(x)$ так же, как в лемме 8. Лемма доказана.

Частичньм обобщением леммы (3.9) из [5, с. 514] является

Лемма 11. Пусть $F(x)$ - функция Римана тригонометрического ряда ви$\partial a(1),(2) u$

$$
\underline{D}^{2} F(x) \geqslant f(x) \quad \text { на } \quad(a, b) \backslash E,
$$

əде $E=\bigcup_{k=1}^{\infty} E_{k}, E_{k}$ являются $H^{\left(s_{k}\right)}$-мнолсествами для некоторых $s_{k}$, а $f(x)$ - интегрируемая на $(a, b)$ функиия такая, что $f(x) \neq-\infty, x \in(a, b)$. Тогда функиия

$$
G(x)=F(x)-\int_{a}^{x} d y \int_{a}^{y} f(t) d t
$$

випукла на $(a, b)$.

ДокАЗАТЕЛЬСтво. Пусть сначала $(a, b)=(0,2 \pi)$. Известно, что для интегрируемой функции $f(x)$ сушествует последовательность минорантных функций $u_{n}(x)$ таких, что $u_{n}(x)$ абсолютно непрерьвны,

$$
\left|u_{n}(x)-\int_{0}^{x} f(t) d t\right|<\frac{1}{n}, \quad x \in(0,2 \pi), \quad n=1,2,3, \ldots,
$$

и $\bar{D} u_{n}(x) \leqslant f(x)$ там, где $f(x)$ конечна, $\bar{D} u_{n}(x) \neq+\infty$. Здесь

$$
\bar{D} u(x)=\varlimsup_{h \rightarrow 0} \frac{u(x+h)-u(x)}{h}
$$

- верхнее производное число функции $u(x)$.

$$
\text { Положим } U_{n}(x)=\int_{0}^{x} u_{n}(t) d t \text {. }
$$


Нетрудно видеть (см., например, [1, с. 785]), что

$$
\bar{D}^{2} U_{n}(x) \leqslant \bar{D} u_{n}(x) .
$$

Тогда

$$
\bar{D}^{2} U_{n}(x) \leqslant \bar{D} u_{n}(x) \leqslant f(x) \leqslant \underline{D}^{2} F(x) \text { на }(0,2 \pi) \backslash E .
$$

Отсюда следует, что

$$
\underline{D}^{2} F(x) \geqslant \bar{D}^{2} U_{n}(x), \quad x \in(0,2 \pi) \backslash E,
$$

причем $\bar{D}^{2} U_{n}(x) \neq+\infty$, a $\underline{D}^{2} F(x) \neq-\infty$ на $(0,2 \pi) \backslash E$. Но тогда

$$
\underline{D}^{2}\left(F(x)-U_{n}(x)\right) \geqslant \underline{D}^{2} F(x)-\bar{D}^{2} U_{n}(x) \geqslant 0, \quad x \in(0,2 \pi) \backslash E
$$

(так как $\underline{D}^{2} F(x)$ и $\bar{D}^{2} U_{n}(x)$ не могут быть бесконечностями одного знака).

Заметим, что $U_{n}(x)$ является функцией Римана ряда Фурье функции $u_{n}^{\prime}(x)$. Следовательно, $F(x)-U_{n}(x)$ - функция Римана ряда вида $(1),(2)$. Отсюда и из леммы 10 следует, что функция $F(x)-U_{n}(x)$ вьпукла на $(0,2 \pi)$. При $n \rightarrow \infty$ последовательность функций $F(x)-U_{n}(x)$ сходится к

$$
G(x)=F(x)-\int_{0}^{x} d y \int_{0}^{y} f(t) d t
$$

поэтому $G(x)$ тоже выпукла на $(0,2 \pi)$.

Рассмотрим теперь произвольный интервал $(a, b) \subset(0,2 \pi)$. Для каждого натурального $n$ определим функцию $\lambda_{n}(x)$, равную нулю на $[0,2 \pi) \backslash(a, b)$, равную единице на $(a+1 / n, b-1 / n)$, неотрицательную на $(a, b)$ и имеюшую три непрерывные производные. Пусть ряд $\sigma_{n}$ есть формальное произведение ряда Фурье функции $\lambda_{n}(x)$ и ряда $S$, а $\Phi_{n}(x)$ - функция Римана ряда $\sigma_{n}$. Положим, далее,

$$
f_{n}(x)= \begin{cases}\lambda_{n}(x) f(x), & x \in(a, b) \\ 0, & x \in[0,2 \pi) \backslash(a, b) .\end{cases}
$$

В силу леммы 3 и условия теоремы

$$
\underline{D}^{2} \Phi_{n}(x)=\lambda_{n}(x) \underline{D}^{2} F(x) \geqslant f_{n}(x), \quad x \in(0,2 \pi) \backslash E .
$$

Тогда по доказанному для интервала $(0,2 \pi)$ отсюда следует, что функция

$$
\Phi_{n}(x)-\int_{0}^{x} d y \int_{0}^{y} f_{n}(t) d t=\Phi_{n}(x)-\int_{a}^{x} d y \int_{a}^{y} f_{n}(t) d t
$$

вьпукла на $(0,2 \pi)$.

Как легко видеть, для $x \in(a, b)$

$$
\lim _{n \rightarrow \infty} \int_{a}^{x} d y \int_{a}^{y} f_{n}(t) d t=\int_{a}^{x} d y \int_{a}^{y} f(t) d t
$$


Так как ряд $\sigma_{n}-\lambda_{n}(x) \cdot S$ сходится к нулю на $[0,2 \pi)$ (см. (16)), а на интервале $(a+1 / n, b-1 / n)$ это означает, что ряд $\sigma_{n}-S$ сходится к нулю, то разность функций Римана $\Phi_{n}(x)-F(x)$ линейна на $(a+1 / n, b-1 / n)$. Следовательно, функция

$$
G_{n}(x)=F(x)-\int_{a}^{x} d y \int_{a}^{y} f_{n}(t) d t
$$

выпукла на $(a+1 / n, b-1 / n)$. Зафиксируем натуральное $N$. Тогда функция

$$
F(x)-\int_{a}^{x} d y \int_{a}^{y} f(t) d t
$$

вьпукла на $(a+1 / N, b-1 / N)$ как предел выпуклых функций $G_{n}(x)$, и в силу произвольности $N$ вьпукла на $(a, b)$. Лемма доказана.

$\mathrm{C}$ помощью леммы 11 может быть доказана напоминающая теорему $\mathrm{A}$

Теорема 5. Пусть $F(x)$ - функиия Римана ряда $S$ вида (1), (2). Если

$$
D^{2} F(x)=f(x), \quad x \in(a, b) \backslash E,
$$

где $E=\bigcup_{k=1}^{\infty} E_{k}, E_{k}$ являются $H^{\left(s_{k}\right)}$-множествами, а $f(x)$ - интегрируемая функция, конечная на $(a, b) \backslash E$, то

$$
F(x)=\int_{a}^{x} d y \int_{a}^{y} f(t) d t+A x+B
$$

ДокАЗАтЕльство. Из неравенства $\underline{D}^{2} F(x) \geqslant f(x), x \in(a, b) \backslash E$, в силу леммы 11 следует, что функция

$$
G(x)=F(x)-\int_{a}^{x} d y \int_{a}^{y} f(t) d t
$$

вьпукла на $(a, b)$.

Полагая $F_{1}(x)=-F(x), f_{1}(x)=-f(x)$, неравенство

$$
\bar{D}^{2} F(x) \leqslant f(x), \quad x \in(a, b) \backslash E,
$$

можно переписать в виде

$$
\bar{D}^{2}\left(-F_{1}(x)\right) \leqslant-f_{1}(x), \quad x \in(a, b) \backslash E,
$$

или

$$
\underline{D}^{2} F_{1}(x) \geqslant f_{1}(x), \quad x \in(a, b) \backslash E .
$$

Отсюда в силу леммы 11 следует, что функция

$$
G_{1}(x)=F_{1}(x)-\int_{a}^{x} d y \int_{a}^{y} f_{1}(t) d t=-\left(F(x)-\int_{a}^{x} d y \int_{a}^{y} f(t) d t\right)=-G(x)
$$

вьпукла, а $G(x)$ вогнута. Следовательно, $G(x)$ линейна, т.е.

$$
F(x)=\int_{a}^{x} d y \int_{a}^{y} f(t) d t+A x+B .
$$

Теорема доказана.

Теперь может быть доказана являющаяся обобщением теоремы 2 Валле-Пуссена (с условием (10)). 
Теорема 6. Пусть $S$ - ряд вида (1), (2), $F(x)$ - функиия Римана ряда $S$, $f$ - конечная интегрируемая функиия на $[0,2 \pi) \backslash E, E \subset[0,2 \pi), E=E_{0} \cup$ $\bigcup_{k=1}^{\infty} E_{k}$, где $E_{0}$ - замкнутое множество единственности, а $E_{k}$ являются $H^{\left(s_{k}\right)}$-множествами для некоторых $s_{k}(k=1,2,3, \ldots)$. Если для ряда $S$ выполняется условие

$$
D^{2} F(x)=f(x) \quad \text { для } \quad x \in[0,2 \pi) \backslash E,
$$

то $S$ есть ряд Фурье функции $f(x)$.

ДоКАЗАТЕЛЬСтво. Пусть $\left(a_{i}, b_{i}\right)$ - смежные интервалы к множеству $E_{0}$. Тогда на каждом таком интервале условие (21) выполняется для $x \in\left(a_{i}, b_{i}\right) \backslash \bigcup_{k=1}^{\infty} E_{k}$. В силу теоремы 5

$$
F(x)=\int_{a_{i}}^{x} d y \int_{a_{i}}^{y} f(t) d t+A_{i} x+B_{i} \text { для } x \in\left(a_{i}, b_{i}\right) .
$$

Сравним $F(x)$ с функцией Римана $\Phi(x)$ ряда Фурье $S(f)$ функции $f(x)$. Известно, что

$$
\Phi(x)=\int_{0}^{x} d y \int_{0}^{y} f(t) d t+A x+B .
$$

Следовательно, $F(x)-\Phi(x)$ линейна на интервалах $\left(a_{i}, b_{i}\right)$,

$$
D^{2}(F(x)-\Phi(x))=0 \quad \text { для } x \in\left(a_{i}, b_{i}\right), \quad i=1,2,3, \ldots
$$

Это означает, что ряд $S-S(f)$ суммируется методом Римана к нулю на $(0,2 \pi) \backslash E_{0}$. Но множество $E_{0}$ как замкнутое $\mathscr{U}$-множество является также $\mathscr{U}(R)$-множеством. Следовательно, $S=S(f)$. Теорема доказана.

Из теоремы 6 следует обобшение теоремы 1 Валле-Пуссена

ТЕОРема 7. Если тригонометрический ряд сходится к конечной интегрируемой функиии всюду на $[0,2 \pi)$ за исключением точек множества $E=E_{0} \cup$ $\bigcup_{k=1}^{\infty} E_{k}$, әде $E_{0}$ - замкнутое множество единственности, а $E_{k}$ являются $H^{\left(s_{k}\right)}$-множсествами для некоторых $s_{k}(k=1,2,3, \ldots)$, то он является рядом Фурье этой функиии.

ДоКАЗАТЕЛЬСТво. Из сходимости тригонометрического ряда почти всюду к конечньм значениям следует в силу теоремы Кантора-Лебега, что коэффициенты ряда стремятся к нулю. Тогда для данного ряда выполняется условие (21) теоремы 6 и, следовательно, он является рядом Фурье своей суммы. Теорема доказана.

Поведение приведенного ядра $R$-нуль-ряда уточняет

Лемма 12. Пусть $S$ - нуль-ряд для метода Римана, $F(x)$ - функиия Римана ряда $S, E \subset[0,2 \pi), E=\bigcup_{k=1}^{\infty} E_{k}$, где $E_{k}$ являются $H^{\left(s_{k}\right)}$-множествами. Пусть $\mathscr{N}_{-}=\left\{x: \underline{D}^{2} F(x)=-\infty\right\} \neq \varnothing$. Тогда и $\mathscr{N}_{-} \backslash E \neq \varnothing$.

Аналогичное утверждение справедливо для $\mathscr{N}_{+}=\left\{x: \bar{D}^{2} F(x)=+\infty\right\}$. 
ДокАЗАТЕЛЬСтво. Так как $S$ является $R$-нуль-рядом, то $D^{2} F(x)=0$ почти всюду. Допустим, что $\mathscr{N}_{-} \backslash E=\varnothing$. Положим $f(x)=\underline{D}^{2} F(x)$. Тогда

$$
\underline{D}^{2} F(x)=f(x)>-\infty \text { для } x \in[0,2 \pi) \backslash E .
$$

В силу леммы 11 функция

$$
F(x)-\int_{0}^{x} d y \int_{0}^{y} f(t) d t=F(x)
$$

вьпукла на $[0,2 \pi)$ и, следовательно, $\underline{D}^{2} F(x) \geqslant 0$ на $[0,2 \pi)$, что противоречит условию $\mathscr{N}_{-} \neq \varnothing$. Поэтому $\mathscr{N}_{-} \backslash E \neq \varnothing$. Случай множества $\mathscr{N}_{+}$сводится к предыдушему рассмотрением ряда $-S$. Лемма доказана.

Известна следующая (см. [5, с. 515])

ТЕОРЕМа БАНАХА-ШТЕЙНГАУЗА. Если тригонометрический ряд $S$ сходится всюду к конечной функиии $f(x)$, причем $f(x) \geqslant g(x)$, где $g(x)$ интегрируема, то $f(x)$ тоже интегрируема и $S$ есть ряд Фурье своей суммы.

Теорема остается справедливой, если сходимость и неравенство выполняются всюду за исключением счетного множества точек. К этому кругу вопросов относится также

Теорема В. А. Скворцова [6]. Если тригонометрический ряд (1), (2) суммируем методом Римана вне замкнутого $H$-множества к конечной функиии $f(x)$, и если $f(x) \geqslant g(x)$, где $g(x)$ интегрируема на $[0,2 \pi)$, то $f(x)$ интегрируема на $[0,2 \pi)$, и ряд есть ряд Фурье функции $f(x)$.

Обобшением этих теорем является

Tеорема 8. Пусть $S$ - ряд вида (1), (2), $F(x)$ - функция Римана ряда $S$, $D^{2} F(x)=f(x)$ конечна на $[0,2 \pi) \backslash E u$

$$
D^{2} F(x) \geqslant g(x) \quad \text { на } \quad[0,2 \pi) \backslash E,
$$

где $g(x)$ - интегрируемая функция, а $E=\bigcup_{k=1}^{\infty} E_{k}$, где $E_{k}$ являются $H^{\left(s_{k}\right)}$-множествами. Тогда $S$ является рядом Фурье.

ДоКАЗАТЕЛЬСтво. В силу леммы 11 из (22) следует, что функция

$$
G(x)=F(x)-\int_{0}^{x} d y \int_{0}^{y} g(t) d t
$$

выпукла на $(0,2 \pi)$, значит, $\underline{D}^{2} G(x) \geqslant 0$ на $(0,2 \pi)$. Можем считать, что $g(x)$ - $2 \pi$-периодическая функция. Тогда неравенство $\underline{D}^{2} G(x) \geqslant 0$ выполняется на $(-\varepsilon, 0) \cup(0,2 \pi) \cup(2 \pi, 2 \pi+\varepsilon)$ для $0<\varepsilon<2 \pi$. Но в точках $x=0$ и $x=2 \pi$ функция $G(x)$ гладкая, так как $F(x)$ - гладкая функция и $\int_{0}^{x} d y \int_{0}^{y} g(t) d t$ тоже гладкая функция. Отсюда следует, что $G(x)$ выпукла на $(-\varepsilon, 2 \pi+\varepsilon)$ и $D^{2} G(x)$ почти всюду существует и интегрируема на $(0,2 \pi)$. Тогда $D^{2} F(x)$ тоже интегрируема на $(0,2 \pi)$. Отсюда в силу теоремы 6 следует, что $S$ есть ряд Фурье функции $f(x)$. Теорема доказана.

В частности, из теоремы 8 следует, что если сумма тригонометрического ряда конечна и неотрицательна вне множества типа $H_{\sigma}$, то она является интегрируемой функцией, и ряд есть ряд Фурье своей суммы.

Теоремы 1,3 и 7 анонсированы в [7]. 


\section{Список литературы}

1. Бари Н. К. Тригонометрические ряды. М.: Физматгиз, 1961.

2. Carlet C., Debs G. Un résultat sur les ensembles d'unicité du tore // Semin. d'Init. à l'Analyse, G. Choquet-m. Rogalski-Y. Saint Raymond, 24-éme année, 1984-85.

3. Холщевникова H. H. О сумме меньше континуума замкнутых $U$-множеств // Вестник МГУ. Сер. матем., мех. 1981. №1. С. 51-55.

4. Пятецкий-Шапиро И. И. К проблеме единственности разложения функций в тригонометрический ряд // Ученые записки МГУ. Математика. 1952. Вып. 155. Т. 5. С. 54-72.

5. Зигмунд А. Тригонометрические ряды. Т. 1. М.: Мир, 1965.

6. Скворцов B. A. О тригонометрических рядах, сходящихся к неотрицательным функциям // Вестник МГУ. Сер. матем., мех. 1962. № 5. С. 3-10.

7. Холщевникова H. H. K теореме Валле-Пуссена о единственности представления // Международная конференция "Функциональные пространства, теория приближений, нелинейньй анализ" (апрель-май 1995 г.). Тезисы докладов. М.: Изд-во ПАИМС, 1995. C. 288 .

Московский государственньй

технологический университет "Станкин"
Поступила в редакцию

05.10 .1995 